\title{
Seismic profile solution under conditions of thawing permafrost with technogenic depression
}

\author{
Vlad Trapeznikov ${ }^{1, *}$, and Dmitry Maleev ${ }^{1}$ \\ ${ }^{1}$ Far Eastern State Transport University, 47, Seryshev St, Khabarovsk, 680021, Russian Federation
}

\begin{abstract}
The article given presents the results of geophysical research of the certain types of geodynamical phenomenon, which is generally located on areas with permafrost soil layers. The detailed description to the research methods of refracting interface of the technogenic thawing ground area under the conditions of flooded pressure by refraction and reflection methods is given. Setting of the problem and detection of the nature of the changes in the properties and configuration of the permafrost and thawed soil layers in the grounds of embankment is practically solved by the integrated application of seismic methods that in theory have different kinematics due to propagation of elastic waves and methods of interpretation the seismic profile.
\end{abstract}

\section{Introduction}

The study of the conditions of occurrence, structure and thickness of the overburden is one of the main tasks of seismic exploration. The formation of irregularities in the boundaries of the seismic profile is due to the manifestation of various geodynamic phenomenons, such as karsts, landslides, collapses, subsidence, etc. When solving this problem, it is necessary to give perform a particular seismic method which will compare the boundary conditions with possible use to geophysical model given and also assessing its advantages and disadvantages over alternative methods [1].

The main types of seismic studies used in observing the conditions of such geological type, structure and thickness of frozen soils in the section are refraction and reflection surveys and also vertical electrical sounding (VES) can be implemented.

The leading method in permafrost geophysics is the VES. The study of a horizontally inhomogeneous section by this method entails a number of serious errors that arise in determining the structure of the geoelectric section. For example, erroneous determination of frozen soils in thawed sections, false allocations of inter-permafrost thawed sections in the layer of frozen soils.

Through the refraction method it is possible to monitor the dynamics of the ground and obtain kinematic parameters of the waves with high accuracy. The conditions for the applicability of the refraction method are limited to several parameters of the object of

\footnotetext{
*Corresponding author: tvladis13@gmail.com
} 
investigation: the minor change in velocities, the low-grade slope of the refracting boundary in comparison with the depth of occurrence and its pseudo-horizontal orientation. This method is relevant for angles of inclination of the refracting boundary not exceeding 15-20 degrees [2].

Under conditions of exploitation of the railway track in the cryolithic zone, a thawed soil is being formed in the basement of the soil structure. Uneven thawing of soil and the influence of surface water determines the formation of sharp angles of the refracting boundary with its complex configuration and the formation of depressions. The presence of these factors makes it impossible to study this geodynamic phenomenon using the method stated above.

The reflection method has a number of advantages over the refraction survey, such as high resolution, depth accuracy, the ability to trace layers with a reduced velocity of elastic waves. The application of the reflected wave method in the study of fictitious zones of geophysical environment is the most rational.

Based on seismic survey performed for the roof of permafrost in the conditions of exploitation of railway track of one of the stations of the eastern section of the Baikal/Amur railway, an area survey technique was developed that makes it possible to restore the refractive boundary of the permafrost layer by means of a combination of refracted and reflected wave methods.

The development of a technique based on a combination of refracted and reflected wave methods is aimed at the possibility of processing and interpreting the field seismic data obtained during from the study of technogenic thawing zones. Simplification of the range of equipment and specialized software was used during seismic data processing which surved as the main advantage of the methodology presented.

\section{Development of a geophysical model}

The object of the study is a geophysical model within the railway track of the Shugara station of the Eastern section of the Baikal/Amur railway.

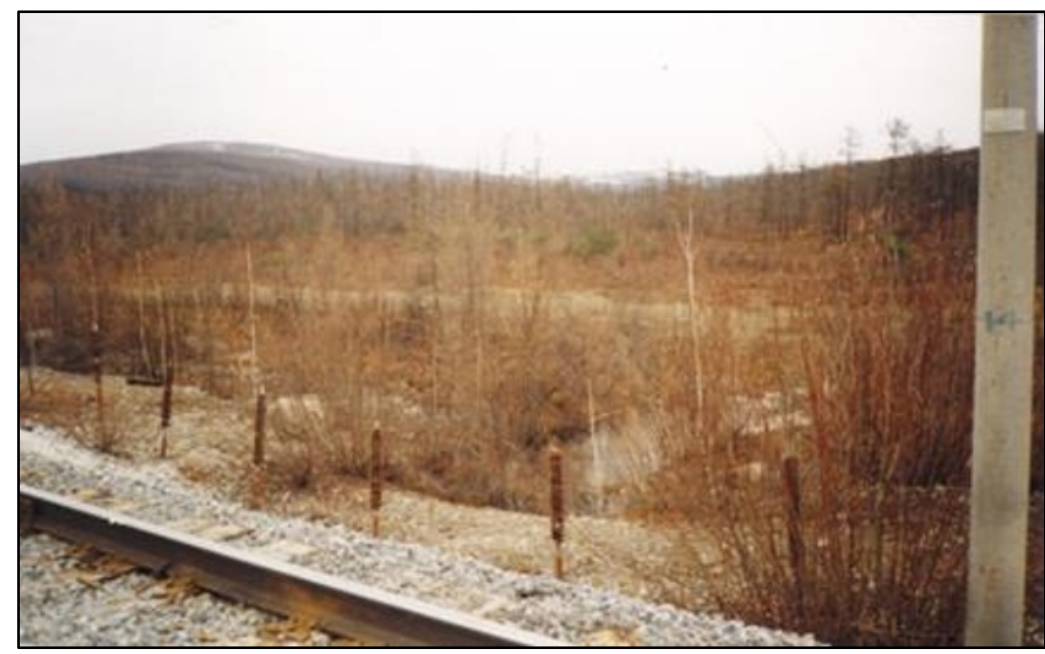

Fig. 1. Drainage roller at the station site (S.M. Zhdanova photo).

Most of the region is located on a relatively large Sredneamurskaya plain, which is a marshy lowland with numerous lakes. Relatively large plains are observed within the Kyndalskaya, Tyrminskaya and Evoron-Chukchagirskaya depressions. They are bogged, 
with numerous thermokarst landslides and oppressed woody vegetation. On the plains are widely developed sphagnum swamps [3].

The frozen zone of the lithosphere in the area with uneven settings of the track is nonuniform permafrost, which is associated with the performance of works during the period of maximum thawing of the permafrost. Under the conditions of the permafrost zone due to the influence of increased circulation of groundwater, formation of a blind thawed section having anthropogenic type was observed at the base of the soil structure. The layer of waterproof is presented by water-saturated quaternary loams, characterized by a high refracted wave velocity $\left(\mathrm{V}_{\mathrm{p}} \approx 4 \mathrm{~km} / \mathrm{s}\right)$.

The surface character of the thawed section in the topography is determined by the presence surface slope of about $1-3 \%$ in the direction perpendicular to the axis of the track. There is non-functioning drainage roller on the upper side of the slope which is the source of surface water inflow to the bottom of the berm.

\section{Seismic data processing}

The geophysical model under investigation is combine. Part of the seismic-geological section outside the zone of water-saturated depression is a horizontally lying flat interface between two homogeneous elastic half-spaces. The seismic refraction profile in this part of the model is assessed by the null-time method and the residual time curve.

The fictitious zone of the model with reduced values of the velocities of elastic waves is formed due to the accumulation of water in the technogenic thawed section in the quasipermanent state. Along with watered depression, the fictitious zone includes the steep sides of the border adjoining it. In the upper half-space, represented by frozen loams, the incident longitudinal wave is observed along the thawed section bead to the media interface. The reflecting boundary is represented by the surface of stationary waters containing watersaturated depression [4].

At the interface between the media, a sharp decrease in the velocities is observed. The velocity of the incident s-wave is less than the velocity of the monotypic transmitted wave. In this case, the longitudinal passing wave travels along the surface of the second medium along its interface. Moreover, the ratio of the angle of incidence of the s-waves incident wave and the critical angle determines the overcritical region which indicates the formation of reflection coefficients and the spreading out of complex numbers in the main math of the profile solution.

At the stage of correlation of target waves, the principle of reciprocity was taken as a criterion of the confirmation of the conclusions. The resulting direct and oncoming hodograph of the head wave are interrelated in the time domain [5].

The propagation of an elastic wave along the slip boundary of a depression in the supercritical domain of the angle of incidence is a consequence of the distortion of the wave pattern. When constructing the difference travel time for spatial interpretation, the $t_{d}$ times serve as reflected "mirror" with respect to the corresponding opposite travel time $\mathrm{T}$ with a preliminary change in its formula:

$$
t_{p}=t_{\text {left }}-t_{\text {right }}+\mathrm{T}
$$




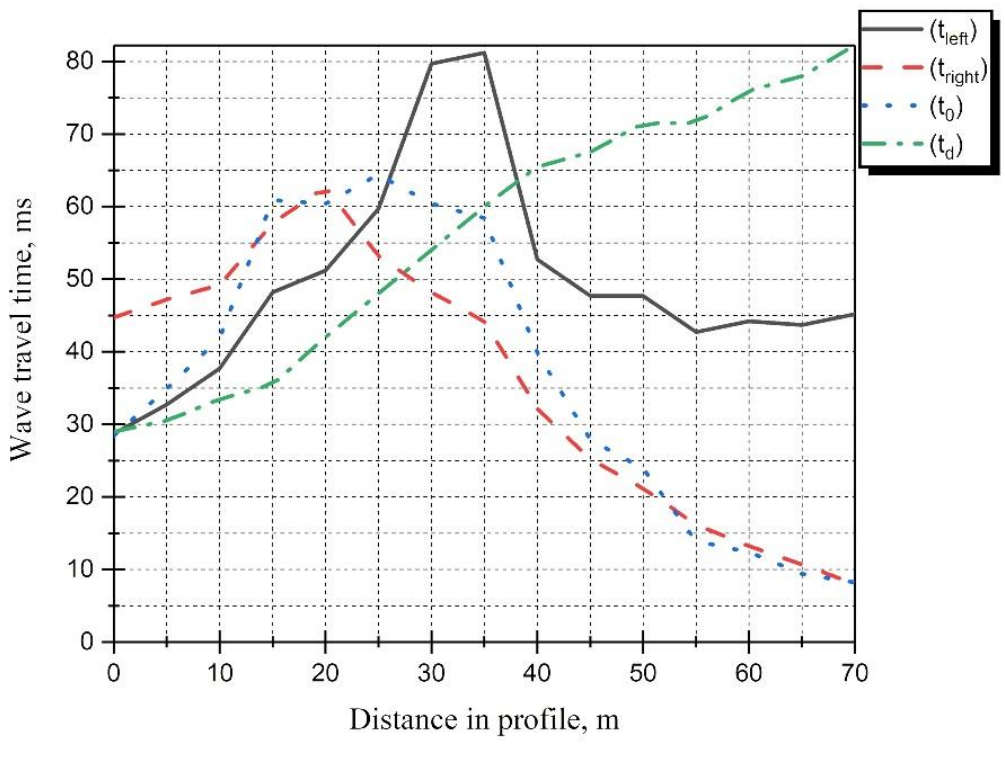

Fig. 2. First arrival curves: $t_{\text {left }}-$ direct; $t_{\text {right }}-$ reversed; $t_{d}-$ difference.

To determine the boundary velocity of the hodograph, the slope angle $\gamma$ of the function $t_{p}=f(x)$ is introduced by the following equation:

$$
V_{d}=2 \cdot \operatorname{tg} \gamma=2 \cdot \frac{\Delta x}{\Delta t_{p}}
$$

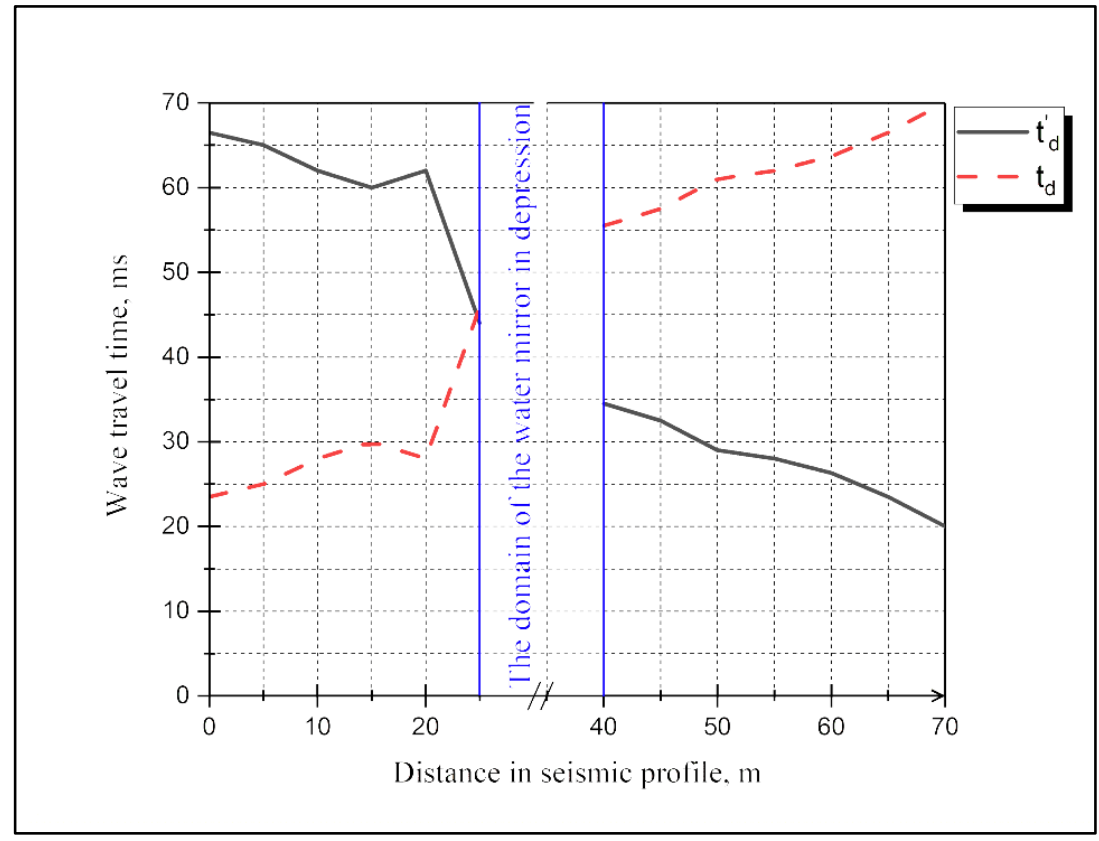

Fig. 3. The slope function of the different-time curve: $a-\operatorname{restored}\left(t_{d}{ }^{\prime}\right) ; b-$ initial $\left(t_{d}\right)$. 
In the case of a mirror transformation of the function with respect to $\mathrm{y}=\mathrm{T}$, a sign change takes place with the tangent of the angle $\gamma$. Numerically, $\gamma_{1}=\gamma_{2}$, but they correspond to different coordinate quarters.

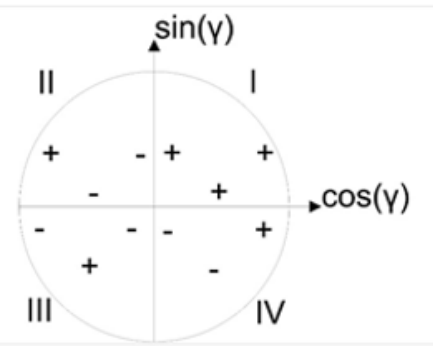

Fig. 4. A unit circle with values of the function.

Table 2. The numerical ranges of the function.

\begin{tabular}{|c|c|c|c|c|}
\hline № & $\gamma$ & $\sin \gamma$ & $\cos \gamma$ & $\operatorname{tg} \gamma$ \\
\hline $\mathrm{t}_{\mathrm{d}}=\mathrm{f}(\mathrm{x})$ & $\gamma_{1}$ & + & - & - \\
\hline $\mathrm{t}_{\mathrm{d}}^{\prime}=\mathrm{f}(\mathrm{x})$ & $\gamma_{2}$ & + & + & + \\
\hline
\end{tabular}

Spatial interpretation of the boundary velocity, i.e. the change of its position from the hypotenuse to the catheter adjacent to the critical angle $i$ is caused by a quarter change at $\tan \gamma$.

Based on this conclusion a distinctive feature of the interpretation of the data of the method null-time method and the difference hodograph under the conditions of the given geophysical model is singled out.

The change in the spatial orientation of the boundary velocity is taken into account by modifying the formula for the coefficient $\mathrm{k}$ :

$$
k=\frac{V_{m d}}{2 \operatorname{cosi}}=\frac{V_{d} V_{m d}}{2 \sqrt{\left(V_{d}^{2}+V_{m d}^{2}\right)}}
$$

In the process of solving of the inverse geophysical problem, the fictitious zone of the model is assessed by the method considered. In the zone of steep beads thawed section, the traveling times of the wave increase significantly, which makes it possible to conclude that the path of the reflected wave must be considered.

Recovered difference hodograph and hodograph null-time method allows to study the occurrence of the conditions of the structure and capacity of permafrost, as well as clearly define the geodynamic phenomenon in the fictitious zone.

\section{Conclusions}

The geophysical model proposed is proofed to be a successful tool for the reverse problem discussed above. The latter has manifested itself by the profile presented in figure 5 . 


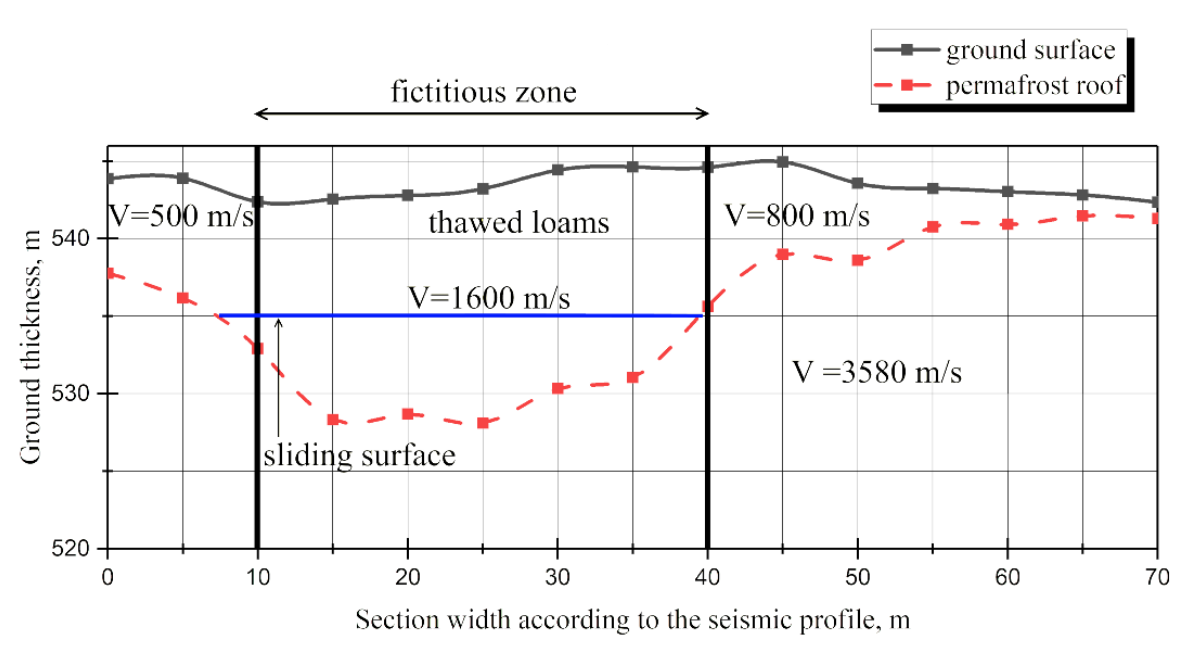

Fig. 5. Seismic profile of the combine geophysical model (the figure shows the s-waves velocities).

The received seismic profile carries the necessary information about the generated anomaly of technogenic origin, sufficient for making further decisions to eliminate the deformations of the railway track operated.

\section{References}

1. V. Trapeznikov, D. Maleev, S. Kvashuk, Designing the development of the regional railway network 5, 111 (2017)

2. F. Lyakhovitsky, V. Khmelevskoy, Z. Yaschenko, Engineering geophysics, 252 (1989)

3. V. Kuznetsov, Russian Journal of Pacific Geology 17, 61 (1998)

4. S. Volokhov, Earth's Cryosphere 11, 35 (2007)

5. V. Bondarev, S. Krylatkov, The theory of travel time curves of reflected waves in the method of multiple overlaps (2001) 